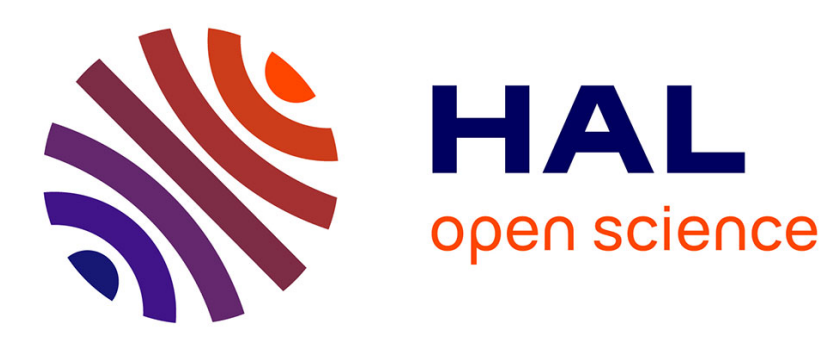

\title{
Modélisation de machines à commutation électronique comportant des matériaux massifs avec circulation de courants de Foucault
}

\author{
B. Davat, M. Lajoie-Mazenc
}

\section{- To cite this version:}

B. Davat, M. Lajoie-Mazenc. Modélisation de machines à commutation électronique comportant des matériaux massifs avec circulation de courants de Foucault. Revue de Physique Appliquée, 1982, 17 (11), pp.745-751. 10.1051/rphysap:019820017011074500 . jpa-00245052

HAL Id: jpa-00245052

https://hal.science/jpa-00245052

Submitted on 1 Jan 1982

HAL is a multi-disciplinary open access archive for the deposit and dissemination of scientific research documents, whether they are published or not. The documents may come from teaching and research institutions in France or abroad, or from public or private research centers.
L'archive ouverte pluridisciplinaire HAL, est destinée au dépôt et à la diffusion de documents scientifiques de niveau recherche, publiés ou non, émanant des établissements d'enseignement et de recherche français ou étrangers, des laboratoires publics ou privés. 


\title{
Modélisation de machines à commutation électronique comportant des matériaux massifs avec circulation de courants de Foucault
}

\author{
B. Davat et M. Lajoie-Mazenc \\ Laboratoire d'Electrotechnique et d'Electronique Industrielle, E.R.A. du C.N.R.S. no 536, \\ 2, rue Camichel, 31071 Toulouse Cedex, France
}

(Reçu le 5 avril 1982, révisé le 12 juillet 1982, accepté le 23 juillet 1982)

\begin{abstract}
Résumé. - Les auteurs présentent une méthode d'étude de machines électriques alimentées en tension par convertisseurs statiques et aux rotors desquelles circulent des courants de Foucault. L'utilisation de la transformation de Laplace permet de résoudre simultanément les équations électriques liant les grandeurs délivrées par le convertisseur et les équations définissant le champ électromagnétique dans la machine. La méthode d'étude proposée est illustrée par l'analyse du fonctionnement d'une machine synchrone à aimants frettés par un cylindre métallique.
\end{abstract}

\begin{abstract}
The authors present a method to study the behaviour of voltage-fed electrical machines where eddy currents are induced in the rotor. The use of the Laplace transformation allows to solve simultaneously the electrical equations which connect the voltages and currents delivered by the converter and the equations which define the electromagnetic field in the machine. The proposed method is illustrated by the analysis of the functionning of a permanent magnet synchronous motor where the magnets are kept in place by a metallic shrink ring.
\end{abstract}

1. Introduction. - Les méthodes d'étude du fonctionnement des machines électriques alimentées par onduleur de tension reposent, en général, sur la connaissance de l'onde de tension délivrée par le convertisseur et d'un système d'équations représentant la machine $[1,2]$.

L'étude du fonctionnement de l'ensemble convertisseur machine s'effectue habituellement en deux étapes bien distinctes :

- une première étape permet de déterminer les caractéristiques de la machine nécessaires à la simulation (inductances, mutuelles...). Cette détermination peut être effectuée analytiquement pour des structures simples, ou numériquement par calcul du champ électromagnétique pour des structures plus complexes $[3,4]$;

- une deuxième étape consiste à résoudre le système d'équations différentielles, représentatif de la machine, mettant en jeu les grandeurs précédemment calculées.

Cette procédure permet l'étude des principaux cas rencontrés dans la pratique (machines à pôles lisses ou saillants avec ou sans amortisseurs) et est également applicable aux machines comportant au rotor des pièces massives où peuvent circuler des courants de Foucault $[5,6,7,8]$.

Cependant, il est intéressant d'obtenir une modélisation directe de l'ensemble convertisseur machine afin d'éviter la décomposition de l'étude en ces deux étapes. Dans cette optique, les auteurs présentent une méthode permettant, après avoir idéaliser les bobinages de la machine, une résolution simultanée des équations électromagnétiques définissant le champ dans la machine et des équations électriques reliant les courants et les tensions d'alimentation. La mise en équation est effectuée analytiquement à l'aide de la transformation de Laplace dans le cas de machines à pôles lisses comportant au rotor des pièces massives en matériau conducteur où peuvent circuler des courants de Foucault.

La méthode de simulation proposée permet, pour des machines dont le rotor est magnétiquement lisse et dont le circuit magnétique n'est pas saturé et pour une alimentation en tension de forme quelconque, de déterminer analytiquement aussi bien le courant d'alimentation que les densités de courants de Foucault circulant dans les parties massives du rotor.

La validité de cette méthode d'étude est illustrée par l'analyse du fonctionnement en régime permanent 
d'une machine synchrone à aimants frettés par un cylindre métallique.

2. Présentation de la méthode d'étude. 2.1 EXPOSÉ DE LA MÉTHODE. - L'étude du fonctionnement d'une machine électrique alimentée par un onduleur de tension repose sur un système d'équations reliant les tensions d'alimentation délivrées par le convertisseur statique et les courants circulant dans les différentes phases de la machine. Dans la phase $n$, cette relation s'écrit :

$v_{\mathrm{s}_{n}}(t)=R_{\mathrm{s}} \cdot i_{\mathrm{s}_{n}}(t)+L_{\mathrm{f}} \frac{\mathrm{d}}{\mathrm{d} t} i_{\mathrm{s}_{n}}(t)+\frac{\mathrm{d}}{\mathrm{d} t} \varphi_{n}(t)+e_{n}(t)$

dans laquelle $R_{\mathrm{s}}, L_{\mathrm{f}}, \varphi_{n}$ et $e_{n}$ représentent respectivement la résistance de l'enroulement, l'inductance de fuite, le flux de réaction d'induit et la f.e.m. aux bornes de l'enroulement.

Pour déterminer, à partir des tensions d'alimentation, le courant $i_{s_{n}}$ circulant dans la machine, il faut connaître le flux $\varphi_{n}$. Ce flux peut se déduire du champ électromagnétique dans la machine. Dans le cas d'une machine comportant des parties conductrices au rotor, où vont circuler des courants de Foucault, lors d'une alimentation non sinusoïdale, la détermination du champ électromagnétique est effectuée en remplaçant les conducteurs de chaque phase par une densité sinusoïdale équivalente de conducteurs. On définit ainsi une densité superficielle de courant à la surface du stator.

La connaissance de la densité du courant permet de déterminer le champ électromagnétique en tout point de la machine. Les équations du champ sont résolues en utilisant le potentiel vecteur. Afin d'obtenir aisément les courants de Foucault induits dans les parties massives, ces différentes grandeurs sont exprimées dans un repère lié au rotor. L'utilisation de la transformation de Laplace conduit à une équation reliant directement le potentiel vecteur dans la machine à la densité de courant circulant à la surface du stator et donc aux courants d'alimentation.

Lorsque le potentiel vecteur à la surface du stator est déterminé analytiquement, c'est le cas en particulier pour les machines à pôles lisses, nous obtenons ainsi une relation permettant de calculer le flux dans les enroulements de la machine. L'équation (1) conduit alors à une expression analytique de la transformée de Laplace du courant d'alimentation. Le courant réel est ensuite obtenu en inversant cette expression à l'aide des algorithmes de la transformée de Fourier rapide.

2.2 RÉSOLUTION DES ÉQUATIONS DU CHAMP ÉLECTROMAGNÉTIQUe. - Pour le calcul du champ électromagnétique dans la machine, on utilise un modèle simplifié de celle-ci. Ce modèle est représenté sur la figure 1. Le référentiel utilisé est lié au rotor. Le rotor, constitué de différentes zones d'épaisseur constante, se déplace par rapport au stator à la vitesse $-\omega / \alpha$,

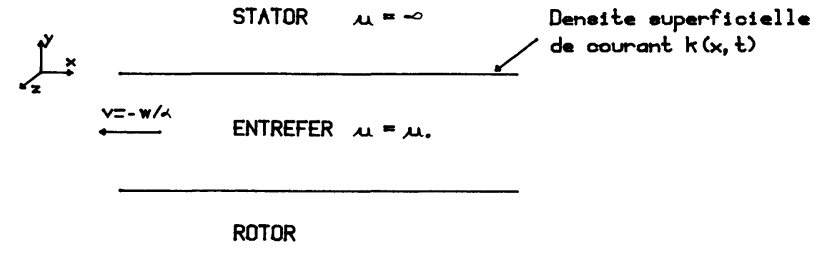

Fig. 1. - Modèle d'étude.

[Model for analysis.]

$\omega$ et $\alpha$ étant la pulsation de rotation et le pas polaire. Pour la phase $n$ et pour une machine triphasée, la densité équivalente de conducteurs est de la forme $k_{0} \sin \left(\alpha x-\omega t+(n-1) \frac{2 \pi}{3}\right)$. La densité de courant à la surface du stator est alors :

$k(x, t)=k_{0} \sum_{n=1}^{3} i_{s_{n}}(t) \cdot \sin \left(\alpha x-\omega t+(n-1) \frac{2 \pi}{3}\right)$.

En négligeant les effets d'extrémité de la machine, on peut supposer que le système de la figure 1 est invariant par translation. Le potentiel vecteur n'a alors qu'une composante selon $z$. Si nous ne tenons pas compte de la saturation, le potentiel vecteur vérifie, en tout point du domaine d'étude, la relation :

$$
\Delta a(x, y, t)=\sigma \mu \frac{\partial a}{\partial t}(x, y, t)
$$

$\sigma$ et $\mu$ étant la conductivité et la perméabilité au point considéré. La perméabilité du stator est supposée infinie, ce qui conduit à la relation suivante entre le potentiel vecteur et la densité superficielle de courant :

$$
\left(\frac{\partial a(x, y, t)}{\partial y}\right)_{y=0}=\mu_{0} k(x, t) .
$$

Pour des conditions initiales nulles des courants d'alimentation et donc de la densité de courant et du potentiel vecteur, la transformation des équations (3) et (4) conduit à :

$$
\begin{aligned}
\Delta A(x, y, p) & =\sigma \mu p A(x, y, p) \\
\left(\frac{\partial A(x, y, p)}{\partial y}\right)_{y=0} & =\mu_{0} K(x, p) \\
A(x, y, p)_{y=-\infty} & =0
\end{aligned}
$$

relations dans lesquelles $A(x, y, p)$ et $K(x, p)$ sont les transformées de Laplace de $a(x, y, t)$ et de $k(x, t)$.

La transformée de Laplace de la densité du courant à la surface du stator est déduite de l'égalité (2) :

$$
\begin{aligned}
& K(x, p)=\frac{k_{0}}{2 j}\left(\sum_{n=1}^{3} I_{s_{n}}(p+j \omega) \times\right. \\
& \times \exp \left[j\left(\alpha x+(n-1) \frac{2 \pi}{3}\right)\right]-\sum_{n=1}^{3} I_{s_{n}}(p-j \omega) \times \\
& \quad \times \exp \left[-j\left(\alpha x+(n-1) \frac{2 \pi}{3}\right)\right] \cdot
\end{aligned}
$$


Dans le cas de machines à pôles lisses, l'expression de la densité de courant permet d'obtenir une solution analytique pour le potentiel vecteur dans la machine $\left({ }^{\mathbf{1}}\right)$. A la surface du stator, le potentiel vecteur est de la forme :

$$
A(x, 0, p)=\Gamma(p) \cdot K(x, p)
$$

Dans cette équation $\Gamma(p)$ dépend de la structure géométrique du rotor et des caractéristiques des différentes zones qui le composent.

2.3 CAlCul DU Flux. - Le flux dans la phase $n$ est obtenu à partir de l'expression du potentiel vecteur à la surface du stator, de la densité de conducteurs de cette phase et de la longueur $l$ de la machine :

$$
\begin{aligned}
\varphi_{n}(t) & =\int_{0}^{2 \pi r} a(x, 0, t) . l \cdot k_{0} \times \\
& \times \sin \left(\alpha x-\omega t+(n-1) \frac{2 \pi}{3}\right) \mathrm{d} x
\end{aligned}
$$

soit pour sa transformée de Laplace $\Phi_{n}(p)$ :

$$
\begin{gathered}
\Phi_{n}(p)=\frac{l k_{0}}{2 j} \int_{0}^{2 \pi r}[A(x, 0, p+j \omega) \times \\
\times \exp \left(j\left(\alpha x+(n-1) \frac{2 \pi}{3}\right)\right)-A(x, 0, p-j \omega) \times \\
\left.\quad \times \exp \left(-j\left(\alpha x+(n-1) \frac{2 \pi}{3}\right)\right)\right] \cdot \mathrm{d} x .
\end{gathered}
$$

L'égalité (6) définissant la densité de courant $K(x, p)$ va permettre d'exprimer ce flux en fonction des courants d'alimentation de la machine :

$$
\begin{aligned}
& \Phi_{n}(p)=\frac{k_{0}^{2} \pi r l}{2}\left[\Gamma(p+j \omega) \sum_{g=1}^{3} I_{\mathrm{s}_{g}}(p) \times\right. \\
& \times \exp \left(-j(g-n) \frac{2 \pi}{3}\right)+\Gamma(p-j \omega) \sum_{g=1}^{3} I_{\mathrm{s}_{g}}(p) \times \\
& \left.\times \exp \left(j(g-n) \frac{2 \pi}{3}\right)\right] \cdot(10)
\end{aligned}
$$

2.4 Calcul du Courant D'alimentation DE LA MACHINE. - La transformation de l'équation (1) permet de calculer le courant d'alimentation. Les conditions initiales sur les courants étant nulles, nous obtenons pour la phase 1 :

$V_{\mathrm{s}_{1}}(p)=\left(R_{\mathrm{s}}+L_{\mathrm{f}} p\right) I_{\mathrm{s}_{1}}(p)+p \Phi_{1}(p)+E_{1}(p)$

et

$$
\begin{aligned}
\Phi_{1}(p)=\frac{l k_{0}^{2} \pi r}{2}\left(\frac{3}{2} I_{\mathrm{s}_{1}}\right. & (p) \cdot S(p)+ \\
& \left.+j \frac{\sqrt{3}}{2}\left(I_{\mathrm{s}_{3}}(p)-I_{\mathrm{s}_{2}}(p)\right) D(p)\right)
\end{aligned}
$$

avec

$$
S(p)=\Gamma(p+j \omega)+\Gamma(p-j \omega)
$$

et

$$
D(p)=\Gamma(p+j \omega)-\Gamma(p-j \omega) .
$$

Le terme $I_{\mathrm{s}_{3}}(p)-I_{\mathrm{s}_{2}}(p)$ est obtenu à partir des tensions aux bornes des autres phases et de l'expression du flux aux bornes des enroulements correspondants :

et

$$
V_{\mathrm{s}_{3}}(p)-V_{\mathrm{s}_{2}}(p)=\left(R_{\mathrm{s}}+L_{\mathrm{f}} p\right)\left(I_{\mathrm{s}_{3}}(p)-I_{\mathrm{s}_{2}}(p)\right)+p\left(\Phi_{3}(p)-\Phi_{2}(p)\right)+E_{3}(p)-E_{2}(p)
$$

$$
\Phi_{3}(p)-\Phi_{2}(p)=\frac{3 l k_{0}^{2} \pi r}{4}\left[\left(I_{\mathrm{s}_{3}}(p)-I_{\mathrm{s}_{2}}(p)\right) S(p)-j \sqrt{3} I_{\mathrm{s}_{1}}(p) D(p)\right]
$$

Soit finalement pour le courant dans la phase 1 :

$$
I_{\mathrm{s}_{1}}(p)=\frac{V_{\mathrm{s}_{1}}(p)-E_{1}(p)+j p \frac{\sqrt{3}}{3} \gamma D(p) \cdot \frac{E_{3}(p)-E_{2}(p)+V_{\mathrm{s}_{2}}(p)-V_{\mathrm{s}_{3}}(p)}{Z(p)}}{Z(p)-\frac{\gamma^{2} p^{2} D^{2}(p)}{Z(p)}}
$$

avec

$$
Z(p)=R_{\mathrm{s}}+L_{\mathrm{f}} p+p \gamma S(p)
$$

et

$$
\gamma=\frac{3 k_{0}^{2} l \pi r}{4}
$$

$\left({ }^{1}\right)$ Voir par exemple le calcul du potentiel vecteur au paragraphe 3 .
Le courant réel s'obtient au moyen de la transformée inverse de Laplace :

$$
i_{\mathrm{s}_{1}}(t)=\frac{1}{2 \pi_{j}} \oint I_{\mathrm{s}_{1}}(p) \mathrm{e}^{p t} \mathrm{~d} p .
$$

Pour un contour d'intégration contenant tous les pôles de $I_{\mathrm{s}_{1}}(p)$, la discrétisation de cette fonction sur le contour permet de calculer $i_{s_{1}}(t)$. Pour une valeur suffisamment grande de $\Omega$, la contribution au calcul 


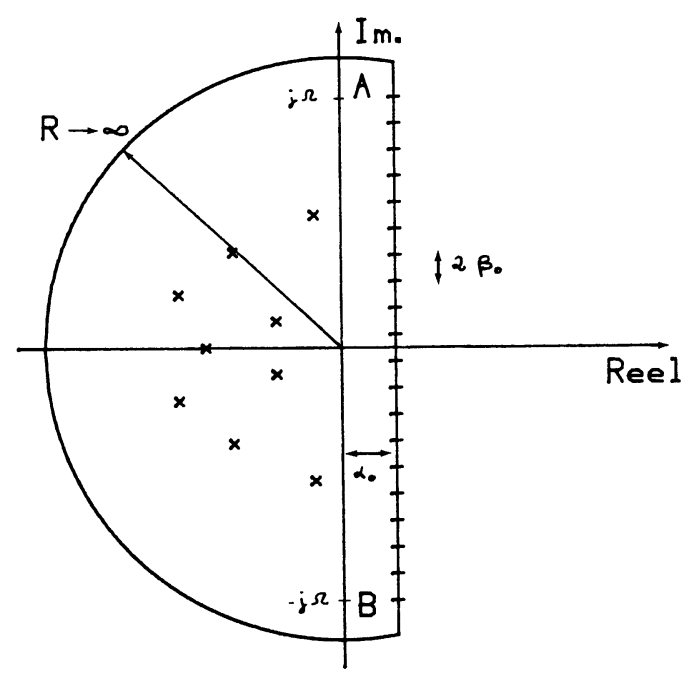

Fig. 2. - Inversion de $I_{\mathbf{s}_{1}}(p)$.

[Inversion of $I_{\mathbf{s}_{1}}(p)$.]

de l'intégrale de la partie du contour autre que le segment $\mathrm{AB}$ est négligeable (Fig. 2). Nous avons alors :

$$
i_{\mathrm{s}_{1}}\left(t_{\mathrm{m}}\right) \simeq \frac{1}{2 \pi_{j}} \sum_{\substack{g=-N \\ g \neq 0}}^{N} I_{\mathrm{s}_{1}}\left(p_{g}\right) \cdot \mathrm{e}^{p_{g} t_{\mathrm{m}}} \cdot j \cdot 2 \cdot \beta_{0}
$$

avec :

$$
\left\{\begin{aligned}
p_{g} & =\alpha_{0}+j(2 g-1) \beta_{0} \\
p_{-g} & =p_{g}^{*}
\end{aligned}\right.
$$

L'utilisation des algorithmes de la transformée de Fourier rapide permet d'accélérer le calcul de $i_{\mathrm{s}_{1}}(t)$ [9].

Les conditions initiales sur le courant d'alimentation étant nulles, les tensions d'alimentation sont imposées aux bornes des enroulements à l'instant $t=0$. Le régime permanent électrique est donc obtenu après un certain nombre de périodes de fonctionnement.

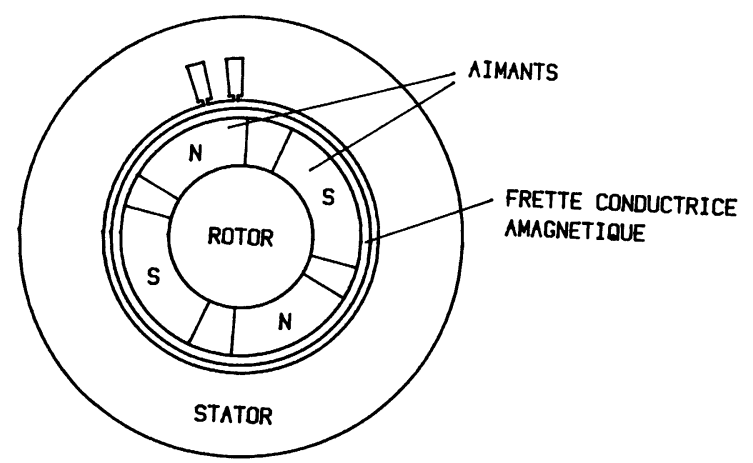

Fig. 3. - Machine synchrone à aimants frettés par un cylindre amagnétique conducteur.

[Permanent magnet synchronous machine where the magnet are kept in place by a conductive non magnetic shrink ring.]

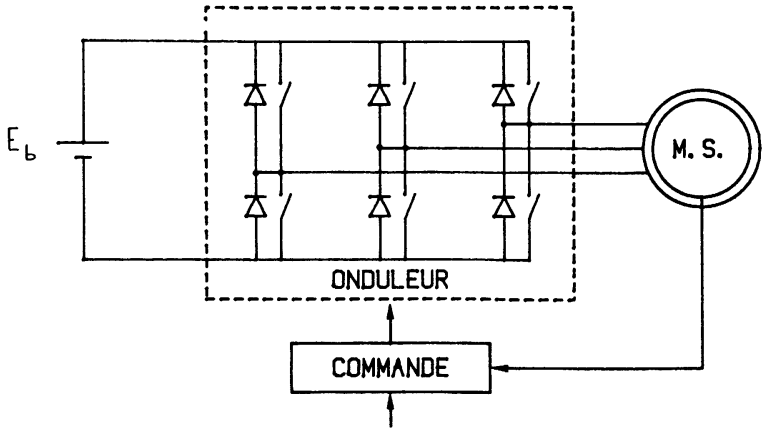

Fig. 4. - Principe de l'alimentation en tension.

[Principle of voltage inverter feeding.]

3. Application à une machine synchrone à aimants. - 3.1 DESCRIPTION DU SYSTÈME ÉTUDIÉ. - Nous allons illustrer la méthode d'étude présentée par l'analyse du fonctionnement en régime permanent d'une machine synchrone à aimants à pôles lisses alimentée par un onduleur de tension.

Le rotor de la machine est constitué d'un noyau magnétique sur lequel sont fixés des aimants ferrites. Les aimants sont tenus par une frette amagnétique en aluminium où les harmoniques de l'alimentation vont induire des courants de Foucault (Fig. 3) [10]. Les caractéristiques du prototype étudié sont données en annexe.

Le principe d'une machine synchrone autopilotée alimentée en tension est défini sur la figure 4. La commande de l'onduleur est élaborée à partir d'un codeur de la position du rotor. De ce fait, le déphasage $\theta$ entre la f.e.m. à vide et le fondamental de la tension d'alimentation est imposé par la commande. Dans la mesure où les commandes des deux interrupteurs situés sur la même branche sont jointes, les tensions aux bornes de la machine sont définies par la seule commande et le choix de la tension batterie $E_{\mathrm{b}}$ et de $\theta$ fixe le point de fonctionnement.

3.2 Calcul du Potentiel vecteur. - Le modèle d'étude, obtenu en développant la machine, est représenté à la figure 5. Nous supposons que le stator est de perméabilité infinie et que le noyau rotorique n'est pas conducteur. Dans un système d'axes lié au rotor, la résolution de l'équation (5) qui définit le potentiel

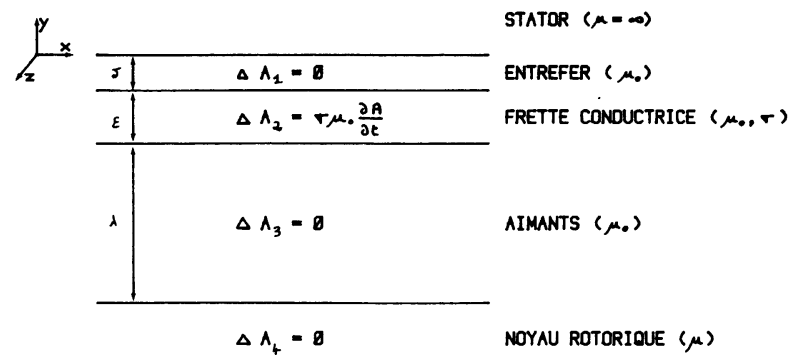

Fig. 5. - Modèle d'étude pour la machine à aimants.

[Model for analysis of the permanent magnet machine.] 
vecteur conduit à l'expression des potentiels vecteurs dans'les différentes zones :

avec :

$$
\begin{aligned}
& A_{1}(x, y, p)=\left(C_{1}(p) \mathrm{e}^{\alpha y}+C_{2}(p) \mathrm{e}^{-x y}\right) \cdot K(x, p) \\
& A_{2}(x, y, p)=\left(C_{3}(p) \mathrm{e}^{b(y+\delta)}+C_{4}(p) \mathrm{e}^{-b(y+\delta)}\right) \cdot K(x, p)
\end{aligned}
$$

$$
\begin{gathered}
b^{2}=\alpha^{2}+\sigma \mu p \\
A_{3}(x, y, p)=\left(C_{5}(p) \mathrm{e}^{\alpha(y+\delta+\varepsilon)}+C_{6}(p) \mathrm{e}^{-\alpha(y+\delta+\varepsilon)}\right) \cdot K(x, p) \\
A_{4}(x, y, p)=C_{7}(p) \mathrm{e}^{\alpha(y+\delta+\varepsilon+\lambda)} \cdot K(x, p) .
\end{gathered}
$$

Les conditions aux limites des différents milieux permettent de déterminer les termes $C_{1}(p) \ldots C_{7}(p)$. Ces conditions s'écrivent :

$$
\begin{aligned}
\left(\frac{\partial A_{1}}{\partial y}\right)_{y=0} & =\mu_{0} K \\
\left(\frac{\partial A_{i}}{\partial x}\right)_{y=y_{i}} & =\left(\frac{\partial A_{i+1}}{\partial x}\right)_{y=y_{i}} \quad i \in\{1,2,3\} \\
\left(\frac{\partial A_{i}}{\partial y}\right)_{y=y_{i}} & =\left(\frac{\partial A_{i+1}}{\partial y}\right)_{y=y_{i}} \quad i \in\{1,2\} \\
\frac{1}{\mu_{0}}\left(\frac{\partial A_{3}}{\partial y}\right)_{y=y_{3}}= & \frac{1}{\mu}\left(\frac{\partial A_{4}}{\partial y}\right)_{y=y_{4}} .
\end{aligned}
$$

On en déduit

$$
\begin{aligned}
& C_{1}(p)=C_{7}(p) \cdot \frac{\mathrm{e}^{\alpha \delta} \alpha}{2 b}\left[\operatorname { s i n h } b \left\{\left(\left(1+\frac{\mu_{0}}{\mu} \frac{b^{2}}{\alpha^{2}}\right) \sinh \alpha \lambda+\left(\frac{\mu_{0}}{\mu}+\frac{b^{2}}{\alpha^{2}}\right) \cosh \alpha \lambda\right)+\right.\right. \\
& \left.+\frac{b}{\alpha} \cosh b \varepsilon\left(1+\frac{\mu_{0}}{\mu}\right)(\sinh \alpha \lambda+\cosh \alpha \lambda)\right] \\
& C_{2}(p)=C_{7}(p) \cdot \frac{\mathrm{e}^{-\alpha \delta} \alpha}{2 b}\left[\sinh b \varepsilon\left(\left(1-\frac{\mu_{0}}{\mu} \frac{b^{2}}{\alpha^{2}}\right) \sinh \alpha \lambda+\left(\frac{\mu_{0}}{\mu}-\frac{b^{2}}{\alpha^{2}}\right) \cosh \alpha \lambda\right)+\right. \\
& \left.+\frac{b}{\alpha} \cosh b \varepsilon\left(-1+\frac{\mu_{0}}{\mu}\right)(\sinh \alpha \lambda-\cosh \alpha \lambda)\right] \\
& C_{3}(p)=C_{7}(p) \cdot \frac{\mathrm{e}^{b \varepsilon} \alpha}{2 b}\left[\sinh \alpha \lambda\left(1+\frac{\mu_{0}}{\mu} \frac{b}{\alpha}\right)+\cosh \alpha \lambda\left(\frac{\mu_{0}}{\mu}+\frac{b}{\alpha}\right)\right] \\
& C_{4}(p)=C_{7}(p) \cdot \frac{\mathrm{e}^{-b \varepsilon} \alpha}{2 b}\left[\sinh \alpha \lambda\left(\frac{\mu_{0}}{\mu} \frac{b}{\alpha}-1\right)-\cosh \alpha \lambda\left(\frac{\mu_{0}}{\mu}-\frac{b}{\alpha}\right)\right] \\
& C_{5}(p)=C_{7}(p) \cdot \frac{\mathrm{e}^{\alpha \lambda}}{2}\left(1+\frac{\mu_{0}}{\mu}\right) \\
& C_{6}(p)=C_{7}(p) \cdot \frac{\mathrm{e}^{-\alpha \lambda}}{2}\left(1-\frac{\mu_{0}}{\mu}\right) \\
& C_{7}(p)=\frac{\frac{b \mu_{0}}{\alpha^{2}}}{\sinh b \varepsilon\left[\sinh \alpha \delta\left(\sinh \alpha \lambda+\frac{\mu_{0}}{\mu} \cosh \alpha \lambda\right)+\frac{b^{2}}{\alpha^{2}} \cosh \alpha \delta\left(\frac{\mu_{0}}{\mu} \sinh \alpha \lambda+\cosh \alpha \lambda\right)\right]+} \\
& +\frac{b}{\alpha} \cosh b \varepsilon\left[\sinh \alpha \delta\left(\frac{\mu_{0}}{\mu} \sinh \alpha \lambda+\cosh \alpha \lambda\right)+\cosh \alpha \delta\left(\sinh \alpha \lambda+\frac{\mu_{0}}{\mu} \cosh \alpha \lambda\right)\right]
\end{aligned}
$$

3.3 Calcul du Courant d'alimentation. - Le terme $\Gamma(p)$ de l'équation (7), nécessaire au calcul du courant d'alimentation, est obtenu à partir de la valeur du potentiel vecteur à la surface du stator : 


$$
\begin{aligned}
\Gamma(p)= & C_{1}(p)+C_{2}(p) \\
& \sinh b \varepsilon\left(b^{2} \sinh \alpha \delta\left(\mu_{0} \sinh \alpha \lambda+\mu \cosh \alpha \lambda\right)+\alpha^{2} \cosh \alpha \delta\left(\mu \sinh \alpha \lambda+\mu_{0} \cosh \alpha \lambda\right)\right)+ \\
=\frac{\mu_{0}}{\alpha} \cdot & \frac{+\alpha b \cosh b \varepsilon\left(\sinh \alpha \delta\left(\mu \sinh \alpha \lambda+\mu_{0} \cosh \alpha \lambda\right)+\cosh \alpha \delta\left(\mu_{0} \sinh \alpha \lambda+\mu \cosh \alpha \lambda\right)\right)}{\sinh b \varepsilon\left(\alpha^{2} \sinh \alpha \delta\left(\mu \sinh \alpha \lambda+\mu_{0} \cosh \alpha \lambda\right)+b^{2} \cosh \alpha \delta\left(\mu_{0} \sinh \alpha \lambda+\mu \cosh \alpha \lambda\right)\right)+} \\
& +\alpha b \cosh b \varepsilon\left(\sinh \alpha \delta\left(\mu_{0} \sinh \alpha \lambda+\mu \cosh \alpha \lambda\right)+\cosh \alpha \delta\left(\mu \sinh \alpha \lambda+\mu_{0} \cosh \alpha \lambda\right)\right)
\end{aligned} .
$$

A partir de cette valeur $\Gamma(p)$, le courant d'alimentation est calculé en utilisant la procédure indiquée au paragraphe 2.4. La figure 6 illustre les résultats obtenus pour un point de fonctionnement $\left(\theta=11^{\circ}\right.$, $E_{\mathrm{b}}=49 \mathrm{~V}$ et $N=2350 \mathrm{t} / \mathrm{min}$.). La photographie correspond aux oscillogrammes de la tension et du courant d'alimentation mesurés sur le montage expérimental. Le courant calculé est présenté au bas de la figure. La comparaison entre les résultats calculés et expérimentaux montre la validité de cette méthode d'étude.
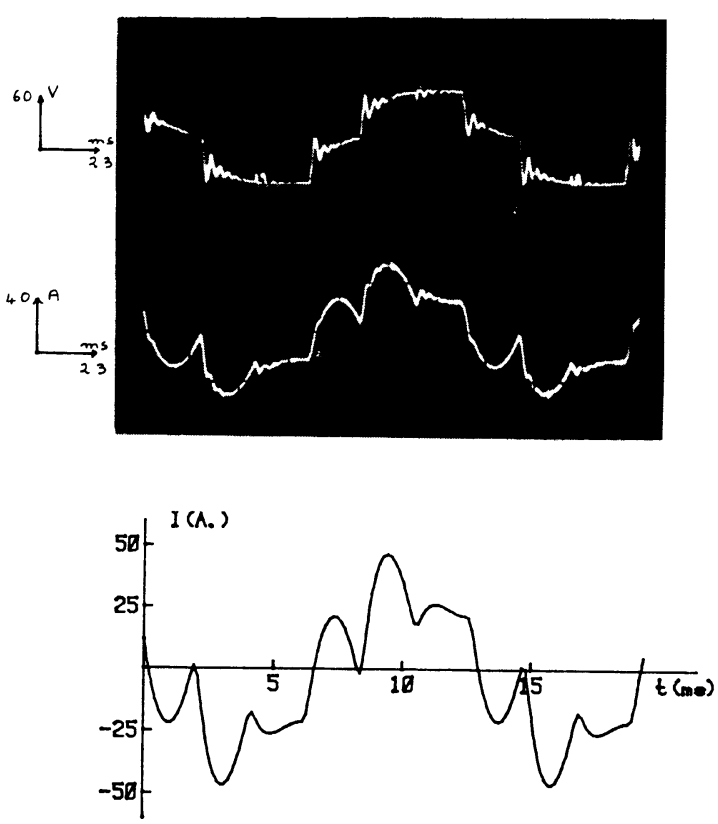

Fig. 6. - Courant et tension d'alimentation.

[Feeding voltage and current.]

3.4 CALCUL DES COURANTS INDUITS DANS LA FRETTE. - La connaissance du potentiel vecteur, dans toutes les parties de la machine, permet de déterminer la densité des courants induits en tout point de la frette. Cette densité s'exprime par :

$$
\begin{aligned}
J(x, y, p)= & -\sigma p A_{2}(x, y, p) \\
= & -\sigma p\left(C_{3}(p) \mathrm{e}^{b(y+\delta)}\right. \\
& \left.+C_{4}(p) \mathrm{e}^{-b(y+\delta)}\right) \cdot K(x, p) .
\end{aligned}
$$

La figure 7 illustre les variations $\mathrm{d}_{u} j(x, y, t)$ en différents points de la frette. Le rotor étant ama-
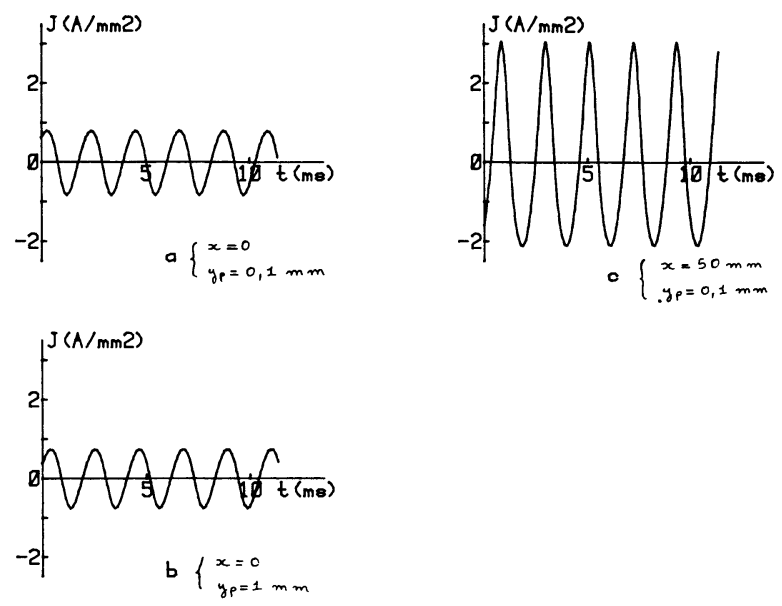

Fig. 7. - Courants induits dans la frette $\left(y_{p}\right.$ : profondeur dans la frette).

[Induced currents in the ring $\left(y_{p}:\right.$ depth in the ring).]

gnétique, pour les fréquences de fonctionnement envisagées les courants induits pénètrent dans toute la frette et leur amplitude varie relativement peu en fonction de la profondeur (Figs. $7 a$ et $7 b$ ). Par contre, pour des points situés à la même profondeur dans la frette, les densités de courants de Foucault peuvent être très différentes (Figs. $7 a$ et $7 c$ ).

4. Conclusion. - La méthode d'étude présentée est illustrée par l'analyse du fonctionnement en régime permanent d'une machine synchrone à aimants, alimentée en tension, dont le rotor est fretté par un cylindre amagnétique conducteur.

La résolution simultanée des équations électriques et électromagnétiques conduit, pour une machine à pôles lisses, à une expression analytique de la transformée de Laplace des courants d'alimentation et des courants induits dans les parties massives conductrices du rotor. Une telle mise en équations pourrait être envisagée pour une machine à pôles saillants en utilisant les méthodes numériques de discrétisation du domaine d'étude. Cependant, cette méthode conduirait à des calculs beaucoup trop importants, les différentes matrices mises en jeu devant être calculées pour chaque valeur $p_{g}$ de l'égalité (16). Pour ce type de machines, une solution totalement numérique avec discrétisation du domaine d'étude et évolution pas à pas du temps semble plus intéressante.

Cette méthode d'étude est donc plus particulièrement destinée aux machines à pôles lisses (machine 
synchrone à aimants frettés, machine asynchrone à rotor massif...), aux rotors desquelles circulent des courants de Foucault induits par l'alimentation électronique. Elle permet d'obtenir une solution analytique du système d'équations électriques et électromagnétiques à partir de la seule connaissance de l'onde de tension délivrée par le convertisseur et des dimensions géométriques de la machine.
5. Annexe. - Caractéristiques du prototype :

Nombre de phases : 3

Nombre de pôles : 4

Diamètre d'alésage : $110 \mathrm{~mm}$

Entrefer : $0,5 \mathrm{~mm}$

Longueur de fer : $140 \mathrm{~mm}$

Epaisseur et nature de la frette : $4,5 \mathrm{~mm}$ en AU4G Epaisseur et nature des aimants : $21 \mathrm{~mm}$ en ferrite.

\section{Bibliographie}

[1] IbRAHIm SAID, A., Etude des caractéristiques d'un dispositif à vitesse variable réalisé à partir d'une machine synchrone alimentée en tension par un convertisseur statique et autopilotée. Thèse de Docteur-Ingénieur, U.P.S. Toulouse (7 mai 1975).

[2] De Fornel, B., Hector, J., Lajoie-Mazenc, M., Trannoy, B., Hybrid simulation of A.C. machines fed by static converters. (ICEM, Bruxelles), 11, 12, 13 septembre 1978, E 6/3-1, E 6/3-10.

[3] Lajoie-MAZENC, M., LeichteR, S., Etude de machines synchrones à aimants alimentées par convertisseur statique en commutation naturelle. (R.G.E.), 3-81, mars 1981, 201-208.

[4] Sabonnadiere, J. C., Meunier, G., Morel, B., Flux : a general interactive finite elements package for 2D electromagnetic fields. IEEE Trans. Magn. 18 (1982) 624, 626.

[5] Davat, B., Lajoie-Mazenc, M., Hector, J., FauCHER, J., Procédé d'analyse des systèmes électromagnétiques avec courants de Foucault : programme DIFIMEDI. Journées S.E.E. « Méthodes de calcul numérique en Electrotechnique ". 5 novembre 1981, Paris.
[6] Davat, B., Rezine, H., Lajoie-Mazenc, M., Eddy currents in solid rotor permanent magnet synchronous motors fed by voltage inverter. Electric Machines and Electromechanics 7 (1982) no 2.

[7] AbDEL RAZEK, AA., Additional losses in solid rotor salient pole synchronous machines associated with static converters. Electric machines and Electromechanics 5 (1980) 101-111.

[8] Bleiss, C. A., Contribution à l'étude de l'alimentation des machines asynchrones à rotor massif non magnétique. Thèse de Docteur ès Sciences Physiques, Paris VI (29 juin 1981).

[9] Wilcox, D. J., Numerical Laplace transformation and inversion. Int. J. Elect. Enging Educ. 15 (1978) 247-265.

[10] Lajoie-Mazenc, M., Leichter, S., Astier, S., Machines à commutation électronique excitées par aimants magnétiquement rigides, Journées SEE « l'Electrotechnique des années 80 », Paris, 3-4-5 décembre 1980. 